\title{
Tuberous sclerosis-associated renal angiomyolipoma: A report of two cases and review of the literature
}

\author{
CANBIN LIN ${ }^{1,2^{*}}$, LU JIN $^{2,3^{*}}$, YU YANG $^{2}$, YU DING $^{2}$, XIONGHUI WU ${ }^{2}$, \\ LIANGCHAO NI ${ }^{2}$, SHANGQI YANG ${ }^{2}$ and YONGQING LAI ${ }^{2}$ \\ ${ }^{1}$ Department of Urology, Shantou University Medical College, Shantou, Guangdong 515041; \\ ${ }^{2}$ Department of Urology, Peking University Shenzhen Hospital, Shenzhen, Guangdong 518036; \\ ${ }^{3}$ Department of Urology, Anhui Medical University, Hefei, Anhui 230032, P.R. China
}

Received November 13, 2016; Accepted July 31, 2017

DOI: $10.3892 /$ mco.2017.1377

\begin{abstract}
In the general population, tuberous sclerosis-associated renal angiomyolipoma (RAML) is a rare benign hamartoma with potentially life-threatening complications and a poor prognosis. The two patients reported in the present study, who were diagnosed with bilateral RAML and spontaneous rupture of the RAML with tuberous sclerosis, presented with flank pain, abdominal pain, hemorrhage, hematuria and multiple lesions. The two cases are representative examples of the disease, and highlight the importance of determining the risk of acute hemorrhage in the early stages, and the significance of timely and proper treatment.
\end{abstract}

\section{Introduction}

Renal angiomyolipoma (RAML) is a type of benign hamartoma that may occur sporadically or be associated with tuberous sclerosis complex (TSC) (1-3). It has been reported that the number of sporadic angiomyolipomas is four times that of the cases associated with TSC $(3,4)$. Generally, $~ 80 \%$ of patients with TSC have hamartoma. However, RAML associated with TSC comprises only $0.3 \%$ of all renal neoplasms (5). In short, RAML is common in patients with TSC, but rare in the general population (2). Notably, TSC-associated RAMLs are typically bilateral and multifocal, larger, and more likely to lead to potentially life-threatening hemorrhage compared with isolated RAML (1).

TSC is a rare autosomal dominant disease with incomplete penetrance; it is characterized by a constellation of findings affecting multiple organ systems, and has a worldwide

Correspondence to: Professor Yongqing Lai, Department of Urology, Peking University Shenzhen Hospital, 1120 Lianhua Road, Shenzhen, Guangdong 518036, P.R. China

E-mail: yqlord@163.com

*Contributed equally

Key words: tuberous sclerosis complex, renal angiomyolipoma, hemorrhage prevalence of $\sim 1 / 6,000$ to $1 / 12,000$ individuals $(2,6-8)$. According to the relevant literature, TSC may be associated with glial tumors, adenoma sebaceum, rhabdomyoma and hamartomatous tumors of the thyroid, retina, liver, pancreas, lung, kidney, adrenals and ovaries. The clinical manifestations of TSC depend on the target organs, such as the brain, skin, kidneys, lungs and heart, or the organs that are oppressed by the expansion of the lesions $(3,9,10)$.

The present study reports two cases of TSC-associated RAML.

\section{Case reports}

Case one. A 31-year-old woman with a chief complaint of left flank pain and abdominal pain of three months duration was admitted to Peking University Shenzhen Hospital (Shenzhen, China) in March 2016. Laboratory examinations yielded the following results: Red blood cell (RBC) count, 3.59x10 $12 / 1$ (normal range, 4.0-5.5 $\times 10^{12} / 1$ ); and hemoglobin (HGB) level, $111 \mathrm{~g} / 1$ (normal range, 120-160 g/l). Subsequently, a contrast-enhanced computed tomography (CE-CT) scan indicated bilateral multiple renal masses with a large hematoma in the left kidney (Fig. 1A), and no enhancement (Fig. 1B). Additionally, magnetic resonance imaging (MRI) of the brain was performed, revealing multiple swellings of the cortex, with increased signal intensity in the left temporal lobe, parietal lobe, occipital lobe, and two frontal lobes, and a small focal ischemia in the right basal ganglion (Fig. 2). The patient was diagnosed with bilateral RAMLs and spontaneous rupture of the left RAML with TSC, which was temporarily managed conservatively without further intervention, as the hemorrhage was controlled by the use of hemostatic drugs; therefore, selective arterial embolization was not performed.

In May 2016, the patient returned to hospital for further treatment. The results of laboratory examinations were as follows: RBC count, 3.77x 10 $12 / 1$; and HGB level, $113 \mathrm{~g} / 1$. In addition, physical examination revealed tenderness and pain on percussion pain over the region of the left kidney. Abdominal palpation revealed a giant mass with tenderness in the left quadrant. Prior to the planned partial nephrectomy, ultrasonography of the urinary system was performed, which revealed bilateral multiple solid lesions; among these masses, 

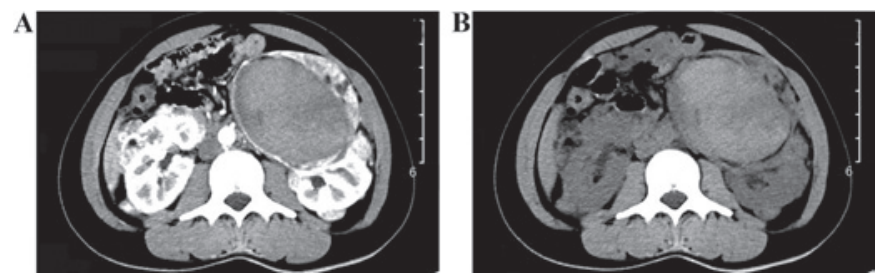

Figure 1. (A) Computed tomography in March 2016 indicated bilateral multiple renal masses with a left massive hematoma; (B) no enhancement was visible.

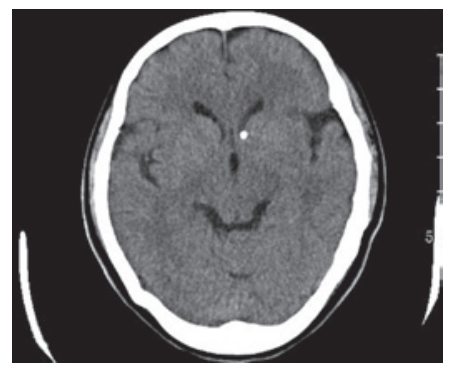

Figure 2. Magnetic resonance imaging of the brain revealed multiple cortical swellings with increased signal intensity in the left temporal lobe, parietal lobe, occipital lobe, and both frontal lobes, and a small focal ischemia in the right basal ganglion.
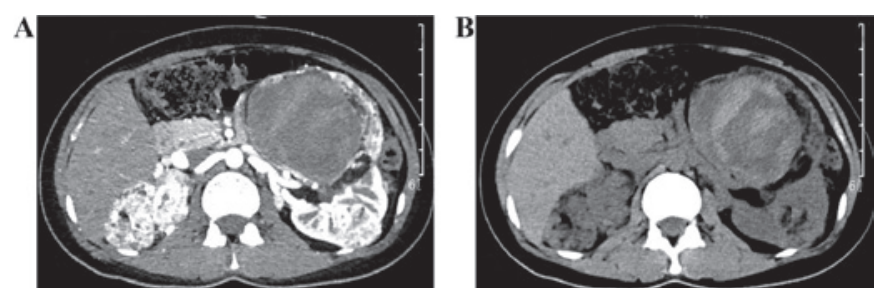

Figure 3. (A) Computed tomography in June 2016 indicated bilateral multiple renal masses with a left massive hematoma; (B) no enhancement was visible.

the largest one was $\sim 8.75 \times 7.95 \mathrm{~cm}$ and located in the left kidney. A further CE-CT scan of the abdomen revealed bilateral multiple renal masses with a massive hematoma in the left kidney (Fig. 3A), with no enhancement (Fig. 3B).

Following the diagnosis of left-sided RAML with TSC, it was determined that the patient required surgical treatment, and a left partial nephrectomy was planned as the tumor size was $>4 \mathrm{~cm}$, in line with the surgical indications. However, during the surgery, the residual kidney tissue was found to be insufficient; therefore, rather than suturing the kidney to retain the residual tissue, a total nephrectomy was carried out. Histological examination indicated a left RAML composed of well-differentiated vessels, smooth muscle and adipose tissue (Fig. 4). To date, the patient has been followed up for $\sim 1$ year post-surgery. The patient recovered well, and no postoperative complications were observed. Urinary ultrasound every 6 months was suggested as follow-up over the next few years.

Case two. In June 2016, a 45-year-old man presented to the Emergency Department of Peking University Shenzhen Hospital with sudden-onset abdominal pain, hematuria and

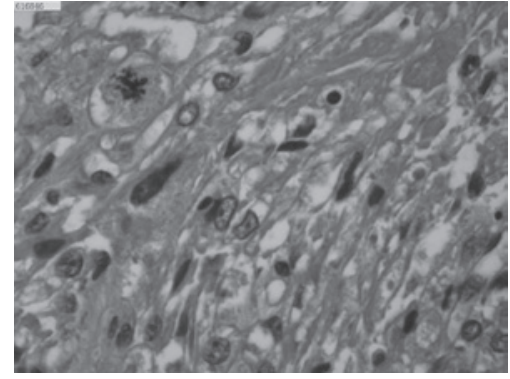

Figure 4. Pathological examination demonstrated that the lesion was composed of well-differentiated adipose tissue, smooth muscle and thick-walled vessels (hematoxylin and eosin staining; magnification, x200).
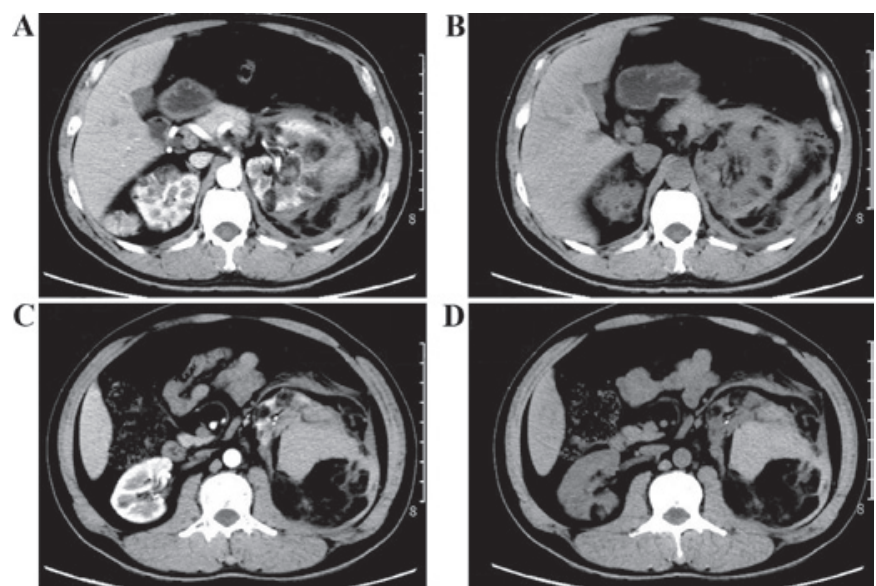

Figure 5. Computed tomography scans. (A) Multiple heterogeneous lesions were detected in the bilateral kidneys with (B) no enhancement. (C) The scans showed a perinephric and subcapsular hematoma secondary to rupture of a left-sided renal angiomyolipoma with (D) no enhancement.

proteinuria. The patient had a history of left lumbar pain of 9 years duration, and his son had previously been diagnosed with bilateral multiple RAML. Laboratory examinations were performed, yielding the following results: RBC count, $4.57 \times 10^{12} / 1$; HGB level, $132 \mathrm{~g} / 1$; white blood cell count, $12.84 \times 10^{9} / 1$ (normal range, $4-10 \times 10^{9} / 1$ ); neutrophilic granulocyte percentage, $82.7 \%$ (normal range, $50-70 \%$ ); urine protein, 2+ (normal, -); urine occult blood, 2+ (normal, -). Emergency ultrasonography was performed, revealing bilateral multiple renal lesions, prompting suspicion for RAML. Among the masses revealed by ultrasound, the largest measured $\sim 11.1 \times 8.6 \mathrm{~cm}$ and was located in the left kidney. Additionally, a CE-CT scan of the abdomen and pelvis was carried out, which showed bilateral renal masses in both kidneys (Fig. 5A), with perinephric and subcapsular hematoma secondary to rupture of the left-sided RAML (Fig. 5C). No enhancement was observed (Fig. 5B and D).

The patient was transferred to the Department of Urology for treatmentofaspontaneousretroperitonealhemorrhage.According to the chief complaint, laboratory examination results, imaging findings and family history of bilateral multiple RAML, the patient was diagnosed with TSC-associated RAML. The patient's condition was managed with conservative treatment, comprising analgesia, hemostasis and fluid infusion; selective arterial embolization was not performed at the time of hospitalization as the hemorrhage had been under control. Partial nephrec- 
tomy was planned for 3 months later as the tumor size of $\sim 10 \mathrm{~cm}$ was in line with the surgical indications. The patient was followed up for $\sim 1$ year. Although a partial nephrectomy was suggested, the patient insisted on conservative treatment, such as use of hemostatic drugs. To date, the patient has only complained of mild flank pain, and no abnormal laboratory examination results have been noted. Partial nephrectomy is still recommended for this patient as it is expected to have a positive effect on preventing rupture of the tumor.

\section{Discussion}

RAMLs are benign hamartomas that are histologically composed of blood vessels, smooth muscle and adipose tissues in variable proportions $(1-3,11)$. For angiomyolipomas in general, $\sim 80 \%$ of cases occur sporadically and $20 \%$ of cases occur in patients with TSC (1). Overall, the number of sporadic RAMLs is four times that of the cases of TSC-associated RAML (3). Furthermore, TSC-associated RAMLs are more likely to be large, multifocal, and complicated by flank pain, abdominal pain, retroperitoneal hemorrhage or hematuria $(1-3,6,11,12)$. TSC-associated RAMLs have a greater risk of hemorrhage compared with sporadic RAMLs (12-14).

As described, TSC-associated RAML is an unusual lesion that behaves like a benign tumor. At present, few cases have been reported that illustrate the potential for life-threatening hemorrhage due to spontaneous rupture of the RAML in patients with TSC; this hemorrhage may occur because, along with the growth of the tumor, the increased blood supply can result in angiectasis and enlargement of arterial aneurysms $(11,12)$. Additionally, multifocal lesions associated with TSC grow rapidly and are more likely to rupture if their size exceeds $4 \mathrm{~cm}(2,5,11)$. Thus, patients with lesions measuring $>4 \mathrm{~cm}$ require proper intervention at the appropriate time in order to avoid the primary complications of retroperitoneal hemorrhage, as the risk of hemorrhage is critical in such patients.

The treatment strategy predominantly depends on the size of the lesion and the presence of symptoms. Surgical treatment for RAML is considered the optimal approach if complications occur, if the size of the tumor reaches $4 \mathrm{~cm}$, or if renal malignancy is suspected. Generally, the indications for intervention include acute hemorrhage and pain, or size $>4 \mathrm{~cm}$ even when asymptomatic $(2,6,8,11)$.

For the two patients described in the current report, rupture of RAML occurred, and the patients presented with flank pain, abdominal pain, hemorrhage and large neoplasms. Typically, the first choice for treatment should be selective embolization. However, in the present cases, hemorrhage was controlled due to the use of hemostatic drugs, so selective arterial embolization was not immediately performed. During the following three months, the two patients complained of only mild flank pain, and had no abnormal laboratory examination results, so selective arterial embolization was still not performed. Eventually, in the fourth month, when bleeding was under control, the tumor size in each case (respectively $\sim 8$ and $\sim 10 \mathrm{~cm}$ ) was in line with the indications for surgery, and surgery was therefore considered. In addition to the typical clinical symptoms, surgical treatment was deemed necessary in the two cases as this is the most effective way to avoid recurrences of tumor rupture and retroperitoneal hemorrhage. Partial nephrectomy was the primary surgical option.

In conclusion, the present case report highlights two important aspects for clinicians. First, in order to avoid potentially life-threatening hemorrhage, the spontaneous rupture of RAML in patients with TSC requires timely and proper intervention by hemostatic drugs or selective arterial embolization. Second, tumors $>4 \mathrm{~cm}$ should be resected to avoid the primary complications of retroperitoneal hemorrhage. It is clear that early detection and diagnosis of the disease can reduce the occurrence of serious complications. In order to ensure timely and proper treatment, urologists should be conscious of the risk of hemorrhage.

\section{Acknowledgements}

The present study was supported by the National Natural Science Foundation of China (grant no. 81101922), Science and Technology Development Fund Project of Shenzhen (grant nos. JCYJ20130402114702124 and JCYJ20150403091443329), the fund of 'San-ming' project of medicine in Shenzhen and the fund of Guangdong Key Medical Subject.

\section{References}

1. Parekh S, Jolapara M, Shah T and Rajpura H: Emergency embolization of actively bleeding renal angiomyolipoma in a patient of tuberous sclerosis. Ren Fail 36: 1114-1118, 2014.

2. Azim A and Rajkumar G: Renal angiomyolipomas in tuberous sclerosis-rare but potentially life-threatening lesions. BMJ Case Rep 2012: pii: bcr2012007720, 2012.

3. Redkar N, Patil MA, Dhakate T and Kolhe P: Tuberous sclerosis complex presenting as bilateral large renal angiomyolipomas. BMJ Case Rep 2012: pii: bcr2012006412, 2012.

4. Kushwaha R, Dhawan I, Arora R, Gupta K and Dhupia JS: Multifocal renal angiomyolipoma presenting as massive intraabdominal hemorrhage. Indian J Pathol Microbiol 53: 340-341, 2010.

5. Moratalla MB: Wunderlich's syndrome due to spontaneous rupture of large bilateral angiomyolipomas. Emerg Med J 26: 72, 2009.

6. Sukumar S, Balagopal Nair T, Mohammed Saheed CS and Sanjay Bhat $\mathrm{H}$ : Bilateral nephron sparing surgeries for multiple renal angiomyolipomas in Bourneville's disease. Int Urol Nephrol 39: 389-391, 2007.

7. Sarraf M, Masoumi A, Castro-Silva FJ, Myers JB, Wilson SS and Schrier RW: A case of tuberous sclerosis complex that progressed to end-stage renal disease. Nat Clin Pract Nephrol 5: 172-176, 2009.

8. Wong IY and Shortliffe LD: The management of renal angiomyolipomas in a patient with tuberous sclerosis. Nat Clin Pract Urol 6: 168-172, 2009.

9. Siroky BJ, Yin H and Bissler JJ: Clinical and molecular insights into tuberous sclerosis complex renal disease. Pediatr Nephrol 26: 839-852, 2011.

10. Dixon BP, Hulbert JC and Bissler JJ: Tuberous sclerosis complex renal disease. Nephron Exp Nephrol 118: e15-e20, 2011.

11. Shen WH, Pan JH, Yan JN, Chen ZW, Zhou ZS, Lu GS and Li WB: Resection of a giant renal angiomyolipoma in a solitary kidney with preoperative arterial embolization. Chin Med J (Engl) 124: 1435-1437, 2011.

12. Granata A, Basile A, Figuera M, Mignani R and Fiore CE: Spontaneous retroperitoneal hemorrhage due to massive rupture of renal angiomyolipoma treated with nephrectomy: An unusual onset of tuberous sclerosis complex. Clin Nephrol 71: 441-444, 2009.

13. Sparks D, Chase D, Thomas D and Arnott J: The Wunderlich's syndrome secondary to massive bilateral angiomyolipomas associated with advanced tuberous sclerosis. Saudi J Kidney Dis Transpl 22: 534-537, 2011.

14. Taviloglu K, Yanar H, Dilege E, Poyanli A, Zorba O, Ertekin C, Guloglu R and Dursun M: Non-traumatic kidney rupture in a tuberosclerosis patient with renal angiomyolipomas. Intern Med J 37: 504-505, 2007. 